\title{
Sunyaev-Zel'dovich distortion from early galactic winds
}

\author{
Subhabrata Majumdar, ${ }^{1,2}$ Biman B. Nath ${ }^{3 \star}$ and Masashi Chiba ${ }^{4}$ \\ ${ }^{1}$ Joint Astronomy Programme, Physics Department, Indian Institute of Science, Bangalore 560012, India \\ ${ }^{2}$ Indian Institute of Astrophysics, Koramangala, Bangalore 560034, India \\ ${ }^{3}$ Raman Research Institute, Bangalore 560080, India \\ ${ }^{4}$ National Astronomical Observatory, Mitaka, Tokyo, Japan
}

Accepted 2000 December 4. Received 2000 November 14; in original form 2000 July 7

\begin{abstract}
A B S T R A C T
We consider the distortion in the cosmic microwave background (CMB) resulting from galactic winds at high redshift. Winds outflowing from galaxies have been hypothesized to be possible sources of metals in the intergalactic medium, which is known to have been enriched to $10^{-2.5} Z_{\odot}$ at $z \sim 3$. We model these winds as functions of mass of the parent galaxy and redshift, assuming that they activate at a common initial redshift, $z_{\text {in }}$, and calculate the mean $y$-distortion and the angular power spectrum of the distortion in the CMB. We find that the thermal Sunyaev-Zel'dovich (SZ) effect resulting from the winds is consistent with previous estimates. The distortion arising from the kinetic SZ (kSZ) effect is, however, found to be more important than the thermal SZ (tSZ) effect. We find that the distortion resulting from galactic winds is an important contribution to the power spectrum of distortion at very small angular scales $\left(l \sim 10^{4}\right)$. We also find that the power spectrum resulting from clustering dominates the Poisson power spectrum for $l \leq(4-5) \times 10^{5}$. We show explicitly how the combined power spectrum from wind dominates over that of clusters at $217 \mathrm{GHz}$, relevant for PLANCK. We also show how these constraints change when the efficiency of the winds is varied.
\end{abstract}

Key words: galaxies: formation - intergalactic medium - cosmic microwave background.

\section{INTRODUCTION}

Recent studies of the epoch of reionization of the Universe offer the following scenario for the first baryonic objects. Although the first objects form at redshifts $z \sim 40$ with masses of order $10^{5} \mathrm{M}_{\odot}$, UV radiation from stars formed in these objects obliterate the molecular hydrogen in other objects in the vicinity, thereby suppressing further formation of objects with masses $M \leq$ $10^{8} \mathrm{M}_{\odot}$ (Haiman, Rees \& Loeb 1997; Ciardi et al. 2000). These objects with masses comparable to local dwarf galaxies appear later, at redshifts $z \lesssim 15$.

These galaxies are thought to have affected the intergalactic medium (IGM) in at least two ways. Along with the radiation from early black holes, they provided the UV radiation needed to reionize the IGM and keep it ionized thereafter. It is also possible that outflows from them provided the metals needed to explain the enrichment of the IGM (Miralda-Escudé \& Rees 1997; Nath \& Trentham 1997). Observations show that the metallicity of the IGM at $z \sim 3$ was $\sim 10^{-2.5} \mathrm{Z}_{\odot}$ (Songaila \& Cowie 1996; Songaila 1997). Although other mechanisms, like galactic mergers, could also have been important in enriching the IGM (Gnedin \& Ostriker 1997), here we consider only galactic winds.

\footnotetext{
${ }^{\star}$ E-mail: biman@ rri.res.in
}

Dwarf starbursting galaxies are expected to produce energetic outflows driven by supernovae and winds from OB stars in them (Larson 1974; Dekel \& Silk 1986). This has been observed both locally (e.g. Heckman 1997) and at high redshift (Franx et al. 1997; Pettini et al. 1998). It is not, however, entirely clear what the efficiency of these outflows is in driving out the gas in the galaxy. Mac Low \& Ferrara (1999) have recently shown that efficient blowaway occurs only in haloes with masses $M \leq 10^{7} \mathrm{M}_{\odot}$, and that a blowout occurs perpendicular to the disc for $10^{8} \leq M \leq 10^{9} \mathrm{M}_{\odot}$, although their simulations assume a disc geometry. However, it is certain that galactic outflows do occur and have important effects on the ambient medium.

Recent studies on galactic outflows at high redshift contend that these winds could have suppressed galaxy formation in the vicinity, for masses $\leq 10^{9} \mathrm{M}_{\odot}$ by mass stripping and gas heating (Scannapieco \& Broadhurst 2001). They assume spherical shells of winds from dwarf spheroidals at high redshift. They also find that these outflows could have distorted the microwave background to an extent that is marginally below the current detection limits, with a mean Compton parameter $\sim 10^{-5}$ depending on the model parameters. Although they did not explicitly calculate the power spectrum of anisotropy in the microwave background, it is clear that distortion from these outflows can be important, and 
deserves detailed calculations and comparison with distortion from other phenomena.

This is what we calculate in this paper. In addition, we take into account the kinetic Sunyaev-Zel'dovich (SZ) effect and the effect of clustering of parent galaxies. The importance of the kinetic SZ (kSZ) effect of outflows - in the case of quasar outflows - has already been shown by Natarajan, Sigurdsson \& Silk (1998) and Natarajan \& Sigurdsson (1999). Here we consider in detail the effect of the outflows from high-redshift dwarf spheroidals on the cosmic microwave background (CMB). We calculate the properties of the outflows, and then the angular power spectrum of the distortions. We consider the effect on the CMB from outflows from a single initial redshift, $z_{\text {in }}$. Finally, we vary this initial redshift and determine the dependence of the distortion on it.

The paper is structured as follows. The next section discusses the characteristics of the outflows, and then in Section 3 we set up an ensemble of these outflows. In Section 4 we introduce the formalism for calculation of the SZ effect from these outflows, and we present the results in Section 5. We end with a discussion of these results in view of several observational constraints.

We assume a flat universe with a cosmological constant, with $\Omega_{0}=0.35, \Omega_{\Lambda}=0.65$ and $h=0.65$, as our fiducial model.

\section{MODELLING THE COSMOLOGICAL OUTFLOWS}

We model the outflows following the approach of Tegmark, Silk \& Evrard (1993, hereafter TSE). They considered the evolution of the blastwaves with an initial energy input (which stops after $t_{\text {burn }} \sim 5 \times 10^{7} \mathrm{yr}$ ), which have thin shells sweeping up most of the ambient IGM gas. The shells are assumed to lose a small fraction $f_{\mathrm{m}}=0.1$ of their mass to the interiors.

We assume, as in TSE, that the total mechanical luminosity of the outflow is $L_{\mathrm{sn}} \sim 1.2\left(M_{\mathrm{b}} / \mathrm{M}_{\odot}\right) \mathrm{L}_{\odot}$, where $M_{\mathrm{b}}$ is the baryonic mass of the parent galaxy. Since the galactic winds last for $t_{\text {burn }} \sim$ $5 \times 10^{7} \mathrm{yr}$, the total energy in the galactic wind is $0.02 \times 0.007 \times$ $M_{\mathrm{b}} c^{2}$, where $M_{\mathrm{b}}$ is the baryonic mass in the galaxy. The factor of 0.02 follows from the observations of Heckman, Armus \& Miley (1990), who show that approximately 2 per cent of the total luminosity of high-redshift starburst galaxies goes into galactic winds. If one assumes the galactic wind to have a constant mechanical luminosity $L_{\mathrm{sn}}$ for the duration of $t_{\text {burn }}$, then TSE show that $L_{\mathrm{sn}} \sim 1.2\left(M_{\mathrm{b}} / \mathrm{M}_{\odot}\right) \mathrm{L}_{\odot}$.

To determine the baryonic fraction, we can either use the baryonic fraction in clusters, or the baryonic fraction of matter in the diffuse IGM, taking $\Omega_{\mathrm{b}}=0.05$. The last fraction, for a lowdensity universe, with $\Omega_{0}=0.35$ as in our model, corresponds to $\sim 0.15$. Also, for clusters, White et al. (1993) has estimated the baryon content to be $M_{\mathrm{b}} / M \geq 0.009+0.05 h^{-3 / 2}$. Adopting the Hubble parameter $h=0.65$ gives the baryon gas mass fraction to be $\sim 0.1$. For these reasons, in this calculation we assume that $M_{\mathrm{b}}=0.1 M$. We also, however, present the final results for the assumption $M_{\mathrm{b}}=0.05 M$, to show the effect of such changes on the final constraints. Since the mechanical luminosity in this model is proportional to $M_{\mathrm{b}}$, this will show the effect of changing the efficiency of winds on our results.

We then numerically solve for the radius and velocity of the shell, and the energy and particle density inside the shell. For the radius and velocity of the shells, we use the equation (1) of TSE, i.e.,

$\ddot{R}=\frac{8 \pi p G}{\Omega_{\mathrm{IGM}} H^{2} R}-\frac{3}{R}(\dot{R}-H R)^{2}-\left(\Omega_{\mathrm{d}}+0.5 \Omega_{\mathrm{IGM}}\right)\left(0.5 H^{2} R\right)$.
Here, $\Omega_{\mathrm{IGM}}$ is the ratio of the density of the intergalactic gas to the critical density, and $\Omega_{\mathrm{d}}$ expresses the total density. The evolution of the total energy inside the shell is given by TSE as

$\frac{\mathrm{d} E_{t}}{\mathrm{~d} t}=L_{\mathrm{sn}}-4 \pi p R^{2} \dot{R}-L_{\mathrm{brem}}-L_{\mathrm{comp}}$.

Here, the first term refers to the energy input from the galaxy, the second term describes the adiabatic loss, the third term describes the energy loss due to bremsstrahlung, and the last term is the energy-loss rate due to inverse Compton scattering off the microwave background photons, which becomes important at high redshifts. $L_{\text {brem }}$ is found to be always much smaller than $L_{\text {comp }}$, as was noted by TSE. We have here neglected the contribution from the somewhat uncertain source of heating to collision of the shell with the IGM from $\left(L_{\text {diss }}\right.$ in TSE) to obtain a conservative estimate of the CMB distortion.

If the shells lose a fraction $f_{\mathrm{m}}$ to the interiors, then the particle density inside the shell of radius $R$ evolves as (assuming a uniform density)

$\frac{\mathrm{d} n}{\mathrm{~d} t}=f_{\mathrm{m}} \frac{\mathrm{d} \rho_{\mathrm{IGM}}}{\mathrm{d} t}+\frac{3}{R} \frac{\mathrm{d} R}{\mathrm{~d} z} f_{\mathrm{m}} \rho_{\mathrm{IGM}}$,

where $\rho_{\text {IGM }}$ is the mass density of the ambient IGM at the given redshift.

For initial conditions, we assume that the initial radius of the shell is the radius of the dwarf spheroidal, for which we use the scaling of the optical size of elliptical galaxies being $=1.2 \times$ $10^{2}\left(M / 10^{12} \mathrm{M}_{\odot}\right)^{0.55} \mathrm{kpc}$ (Saito 1979; Matteucci \& Tornambé 1987). This is a useful relation, although its validity has not been updated with more recent data. Our wind solutions, however, do not depend strongly on the assumption of this initial point, and therefore its exact validity is not relevant here. The initial velocity is expected to be of the order of the thermal velocity of gas heated to $10^{6} \mathrm{~K}$. We assume here that the blastwaves expand into an already ionized medium, and therefore neglect the energy loss due to ionization of the IGM (as considered by TSE). The early supernovae driven winds are expected to expand into an IGM, which has already been ionized by the same massive stars which gave rise to the supernovae later (Ferrara, Pettini \& Shchekinov 2000). We assume that $\Omega_{\mathrm{IGM}}=\Omega_{\mathrm{b}}=0.05$ as constrained by big bang nucleosynthesis (for $h=0.65$ ), since at redshift $z \sim 4-5$ most of the baryons are in the IGM (Weinberg et al. 1997; Rauch et al. 1998).

With the above equations and initial conditions we calculate the wind parameters, for a given initial redshift $z_{\text {in }}$, as functions of the mass of the parent galaxy and redshift, using a fourth-order Runge-Kutta technique. We plot in Fig. 1 the evolution of the radius (in physical coordinates) and the electron temperature for winds out of galaxies of masses $M=10^{9}$ and $10^{10} \mathrm{M}_{\odot}$ for $1+z_{\text {in }}=15$ and 20 . We have checked that our results for radius and $T_{\mathrm{e}}$ match with those of Scannapieco \& Broadhurst (2000) for similar parameters (their fig. 3).

We note here that the uncertainty in the efficiency of mass-loss in galactic outflows, as questioned by Mac Low \& Ferrara (1999), is not relevant here, as we assume that the entire mass in and inside the shell is provided by the ambient IGM. We use only the mechanical energy output of the parent galaxy in our calculations.

\section{ENSEMBLE OF GALACTIC OUTFLOWS}

Although a series of outflows would probably occur in the Universe, for simplification and tractability of the calculation we 


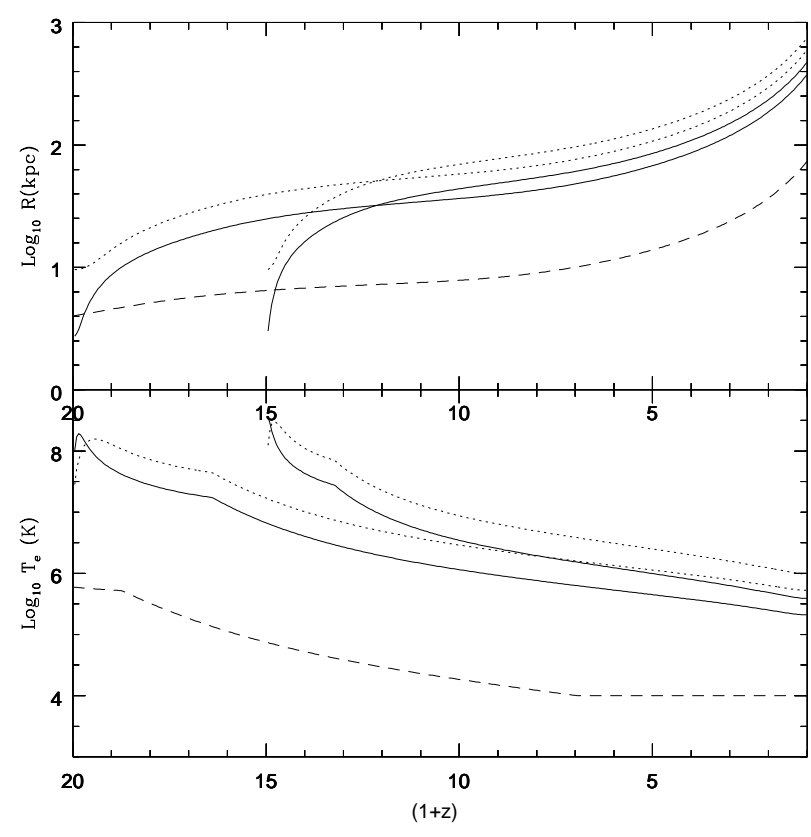

Figure 1. The evolution of radius and electron temperature are shown for $1+z_{\text {in }}=15$ and 20 , and for two masses of galaxies, $M=10^{9} \mathrm{M}_{\odot}$ (solid lines) and $10^{10} \mathrm{M}_{\odot}$ (dotted lines). We also plot with dashed lines the case for wind for $M=5 \times 10^{7} \mathrm{M}_{\odot}$, with $z_{\text {in }}=25$ and the parameters used by Scannapieco \& Broadhurst (2001), for comparison. The temperature is not allowed to fall below $10^{4} \mathrm{~K}$, as in their work, considering the heating due to UV background radiation.

consider the case of outflows originating at a single epoch, at $z_{\text {in }}$. We consider a large range of values for $3 \leq z_{\text {in }}<15$. This assumption of a single epoch can be justified to some extent because of the fact that after the first objects of mass of order $\sim 10^{8} \mathrm{M}_{\odot}$ start shining and outflowing, the resulting UV background radiation and the winds both inhibit the further formation of low-mass objects: photons do this by heating up the IGM gas (Thoul \& Weinberg 1996), and the outflows, by gas stripping (Ferrara et al. 2000). Therefore, if there were widespread outflows associated with the epoch of first luminous objects with masses of order $10^{8} \mathrm{M}_{\odot}$, it could not have lasted a long time.

We use the results of the previous section to set up an ensemble of galactic outflows from galaxies with masses $5 \times 10^{7} \leq M \leq$ $10^{11} \mathrm{M}_{\odot}$, using the abundance of collapsed objects as predicted by a modified version of the Press-Schechter (PS) mass function (Press \& Schechter 1974) given by Sheth \& Tormen (1999), which matches $N$-body simulations well at galactic scales. We assume that the galaxy number density is linearly biased and traces the abundance of collapsed dark matter haloes. The lower limit of mass of parent galaxies is motivated by the fact that the first baryonic objects to shine after the initial pause (due to destruction of molecular hydrogen) have a virial temperature of order $10^{4} \mathrm{~K}$, with corresponding masses of order $5 \times 10^{7} \mathrm{M}_{\odot}$ (Haiman et al. 1997; Scannapieco \& Broadhurst 2001). The upper limit of masses is motivated by the fact that a blowout seems to be inhibited for masses above $10^{12}(1+z)^{-3 / 2} \mathrm{M}_{\odot}$, as reported by Ferrara et al. (2000), although we note that these limits apply strictly to disc galaxies, whereas we consider here dwarf spheroidals as parent galaxies.

The PS mass function essentially calculates the mass function of objects in terms of their collapse redshift $z_{\mathrm{c}}$. In principle, the epoch of galactic winds will occur later, and so, in principle, $z_{\text {in }} \leq z_{\text {col }}$. The fractional difference between these two epochs is, however, extremely small. There are two effects that can make $z_{\text {in }}$ differ from $z_{\mathrm{c}}$ in principle: the extra time for cooling, and then the time for galactic winds. First, the change in the PS formalism due the effect of cooling, from $\left(1+z_{\mathrm{c}}\right)$ to $\left(1+z_{\mathrm{c}}\right)\left(1+M / M_{\mathrm{cool}}\right)^{2 / 3}$ is negligible here, as $M_{\text {cool }} \sim 3.6 \times 10^{11} \mathrm{M}_{\odot}$ (Peacock \& Heavens 1990), larger than the objects considered here. Moreover, the timescale for the galactic wind for dwarf galaxies is much smaller than a Hubble time at $z \sim 15$ (Nath \& Trentham 1997). We therefore assume that $z_{\text {in }}=z_{\mathrm{c}}$.

We use the transfer function of Bardeen et al. (1986), with the shape parameter given by Sugiyama (1995), and the Harrison Zel'dovich primordial spectrum to calculate the matter power spectrum $P_{\mathrm{m}}(k)$. The resulting mass variance $\left(\sigma_{8}\right)$ on scales of $8 h^{-1} \mathrm{Mpc}$ is normalized to 4-year COBE - FIRAS data using the fit given by Bunn \& White (1997). This normalization, unlike that from cluster abundance, is free of uncertainties of physics of cluster formation.

Once the objects are distributed, we then calculate the peculiar velocities with respect to the $\mathrm{CMB}$ frame of reference. The velocities are usually assumed to follow a Gaussian (Bahcall, Cen \& Gramann 1994; Bahcall \& Oh 1996; Moscardini et al. 1996) or Maxwellian distribution (Molnar \& Birkinshaw 2000), which is completely defined by its rms value $\sigma_{v}$. For analytical simplicity, we assume a mean peculiar velocity for all galaxies for a given redshift and cosmology, which is equal to the rms value $\sigma_{v}$ of the distribution. Following Molnar \& Birkinshaw, we can write the cluster peculiar velocity $v_{r}$ expected from a Gaussian initial density field as as Maxwellian distribution with a pdf given by $v_{r}^{2} \exp \left[-v_{r}^{2} / 2 \sigma_{v}(z)^{2}\right]$. The rms peculiar velocity is then given by $\left\langle v^{2}\right\rangle_{R}(z)=H^{2}(z) a^{2}(z) f^{2}\left(\Omega_{0}, \Lambda\right) \sigma_{-1}(R)$, where $a(z)$ is the scalefactor, and $\sigma_{-1}(R)=1 / 2 \pi^{2} \int_{0}^{\infty} P(k) W(k R) \mathrm{d} k$. Here $W_{R}$ is the tophat window function for smoothing. The velocity factor is $f(z) \equiv$ $\mathrm{d} \ln \delta / \mathrm{d} \ln a$ [see Peebles 1980 and Lahav et al. 1991, for a detailed expression of $f(z)$ ]. From averaging over the above Maxwellian pdf, one can get the Maxwellian width given by

$\left\langle v_{r}^{2}\right\rangle=\frac{\int_{0}^{\infty} v^{4} \exp \left[-v_{r}^{2} / 2 \sigma_{v}(z)^{2}\right] \mathrm{d} v}{\int_{0}^{\infty} v^{2} \exp \left[-v_{r}^{2} / 2 \sigma_{v}(z)^{2}\right] \mathrm{d} v}=3 \sigma_{v}^{2}$.

Next, one expresses $\sigma_{v}$ as Normalization $\times[H(z) a(z) f(z)] /$ $[H(0) a(0) f(0)]$. The normalization at $z=0$ is taken to be $v_{r 0}=$ $400 \mathrm{~km} \mathrm{~s}^{-1}$ for flat models with a cosmological constant (Gramann 1998). However, we also show our results with a smaller $v_{r}(z=0)=300 \mathrm{~km} \mathrm{~s}^{-1}$. For the $\Lambda$ CDM model we can write $\sigma_{v}$ explicitly (Molnar \& Birkinshaw 2000) as

$$
\begin{aligned}
\sigma_{v}\left(\Omega_{0}, \Lambda, z\right)= & 400 \mathrm{~km} \mathrm{~s}^{-1} \frac{1+z}{\left[\Omega_{0}(1+z)^{2}+1-\Omega_{0}\right]^{1 / 2}} \frac{D_{\Lambda}\left(\Omega_{0}, 0\right)}{D_{\Lambda}\left(\Omega_{0}, z\right)} \\
& \times\left[\frac{5-3(1+z) D_{\Lambda}\left(\Omega_{0}, z\right)}{5-3 D_{\Lambda}\left(\Omega_{0}, 0\right)}\right] .
\end{aligned}
$$

In the above formula, $D_{\Lambda}\left(\Omega_{0}, z\right)$ is the linear growth factor (see Caroll, Press \& Turner 1992 for an approximate expression for a $\Lambda \mathrm{CDM}$ universe). Given a large number of realizations of a given cosmology, we expect the final result to be close to that obtained from using the mean velocity.

\section{SUNYAEV-ZEL'DOVICH DISTORTION}

The hot gas in the interior of the shells can distort the microwave background, introducing temperature anisotropies, through the 
Sunyaev-Zel'dovich effect (Sunyaev \& Zel'dovich 1972). Scattering of the photons off hot electrons causes the radiation to gain energy. Also, conservation of photons in the scattering results in a systematic shift in photon number from the Rayleigh-Jeans (R-J) to the Wein side of the spectrum. The spatial dependence of the effect is given by the Comptonization parameter $y=$ $2 \int_{0}^{R} \frac{k_{\mathrm{B}} T_{\mathrm{e}}}{m_{\mathrm{e}} c^{2}} \sigma_{\mathrm{T}} n_{\mathrm{e}} \mathrm{d} l$, where $n_{\mathrm{e}}$, and $T_{\mathrm{e}}$ refer to the electron density and temperature inside the shell of radius $R$ (physical size), $\sigma_{\mathrm{T}}$ is the Thomson cross-section, $k_{\mathrm{B}}$ is the Boltzmann constant, and $m_{\mathrm{e}}$ is the electron mass. The thermal SZ (tSZ) effect is characterized by its specific spectral signature. The change of spectral intensity, $I$, along the line of sight is given by $\Delta I_{\text {th }}=y i_{0} g(x)$, with $i_{0}=$ $2\left(k_{\mathrm{b}} T_{0}\right)^{3} /(h c)^{2}$. The spectral form of the tSZ effect is given by

$g(x)=\frac{x^{4} \mathrm{e}^{x}}{\left(\mathrm{e}^{x}-1\right)^{2}}[x \operatorname{coth}(x / 2)-4]$

where $x=h \nu / K_{\mathrm{b}} T_{0}$. In the non-relativistic limit the spectral function is zero at the crossover frequency $x_{0}=3.83$ (i.e., $\nu=$ $217 \mathrm{GHz}$ with $T_{0}=2.726$ ). The frequency dependence of the corresponding temperature change due to the tSZ effect is given by

$\frac{\Delta T}{T_{0}}=[x \operatorname{coth}(x / 2)-4] y$.

If $x \ll 1$ (R-J limit), then $\Delta T / T_{0}=-2 y$, and for $x \gg 1, \Delta T / T_{0}=$ $x^{2} y$. This specific spectral dependence of the tSZ effect can be used to separate it out from the primary anisotropy. The tSZ effect is caused by the random thermal motion of the electrons whose distribution is isotropic (in the wind frame).

An additional source of anisotropy is due to the Doppler effect of the CMB photons if the shell has a peculiar velocity. For a radial peculiar velocity $v_{r}$ of the shell (w.r.t. the Hubble flow), the amplitude of the kinetic SZ (kSZ) effect is given by

$\frac{\delta T}{T}=\frac{v_{r}}{c} \times \int_{-R}^{R} \sigma_{\mathrm{T}} n_{\mathrm{e}} \mathrm{d} l$.

The intensity change due to the peculiar motion is $\Delta I_{\text {kin }}=$ $-\frac{v_{r}}{c} \tau i_{0} h(x)$, where $\tau$ is the cluster optical depth to Compton scattering, and

$h(x)=\frac{x^{4} \mathrm{e}^{x}}{\left(\mathrm{e}^{x}-1\right)^{2}}$.

However, in contrast to with the temperature change due to the $\mathrm{tSZ}$ effect, the kinematic temperature change due to the $\mathrm{kSZ}$ effect is independent of frequency (see Rephaeli 1995). Thus one can utilize the different frequency dependence of temperature anisotropy of the tSZ and $\mathrm{kSZ}$ effects to separate them out in any observation involving multiple frequency.

The ratio of the tSZ effect to the $\mathrm{kSZ}$ effect is then given by $\frac{k_{\mathrm{B}} T_{\mathrm{e}}}{m_{\mathrm{e}} c^{2}} / \frac{2 v_{r}}{c}$. It is generally found that larger structures (like clusters of galaxies), which form later, will have a larger tSZ effect than $\mathrm{kSZ}$ effect, whereas structures going back to high redshifts (like quasar ionized bubbles, black-hole seeded protogalaxies; see Aghanim et al. 1996, Natarajan \& Sigurdsson 1998 and Aghanim, Balland \& Silk 2000) will have the opposite behaviour. CMB distortions due to galactic winds fall into the second category.

For simplicity, we assume that the density and temperature inside the shells are uniform. At high redshifts, this is a realistic approximation as the sound crossing time is less than the Hubble time. At lower redshifts, this approximation admittedly breaks down to some extent.
The fluctuations of the CMB temperature produced by either the tSZ effect or the kSZ effect can be quantified by their spherical harmonic coefficients $a_{l m}$, which can be defined as $\Delta T(\boldsymbol{n})=$ $T_{0}^{-1} \sum_{l m} a_{l m} Y_{l m}(\boldsymbol{n})$. The angular power spectrum of the SZ effect is then given by $C_{l}=\left\langle\left|a_{l m}\right|^{2}\right\rangle$, the brackets denoting an ensemble average. We first consider the objects to be Poisson random distributed, and consider the correlation between them later. The power spectrum for the cosmological distribution of objects can then be written as (Peebles 1980; Cole \& Kaiser 1988)

$C_{l}^{\text {Poisson }}=\int_{0}^{z_{\max }} \mathrm{d} z \frac{\mathrm{d} V(z)}{\mathrm{d} Z} \int_{M_{\min }}^{M_{\max }} \mathrm{d} M \frac{\mathrm{d} n\left(M, z_{\text {in }}\right)}{\mathrm{d} M}\left|y_{l}(M, z)\right|^{2}$,

where $V(z)$ is the comoving volume, and $\mathrm{d} n / \mathrm{d} M$ is the number density of objects. Following Komatsu \& Kitayama (1999), we define the power spectrum of $y$-parameter which is independent of frequency as $C_{l}=C_{l}(x) / g^{2}(x)$, with $g(x)$ given by equation (4) for the tSZ effect. For the kSZ effect $C_{l}=C_{l}(x)$. (Note that $z_{\max } \leq z_{\text {in }}$ in our case.) In our calculation, we use a modification of the Press-Schechter distribution, which provides a good fit to the unconditional halo mass function for different cosmologies in the mass range of our interest (details can be found in Sheth \& Tormen 1999). Since these fluctuations occur at very small angular scales, we can use the small-angle approximation of the Legendre transformation and write $y_{l}$, the angular Fourier transform of $y(\theta)$, as $\left.y_{l}=2 \pi \int_{r_{\text {gal }}}^{r_{\text {wind }}} y(\theta) J_{0}[(l+1 / 2]) \theta\right] \theta \mathrm{d} \theta$. We have used the approximation $\left.P_{l}^{\text {gal }} \approx J_{0}[(l+1 / 2]) \theta\right]$, where $P_{l}$ is a Legendre coefficient, and $J_{0}$ is a Bessel function of first kind and zero order (Peebles 1980; Molnar \& Birkinshaw 2000).

At high redshifts we expect a significant contribution to the anisotropy from correlation among the structures. Following Komatsu \& Kitayama (1999), we estimate the angular power spectrum as

$$
\begin{aligned}
C_{l}^{\text {clustering }}= & \int_{0}^{z_{\max }} \mathrm{d} z \frac{\mathrm{d} V(z)}{\mathrm{d} Z} P_{\mathrm{m}} \\
& \times\left[\int_{M_{\min }}^{M_{\max }} \mathrm{d} M \frac{\mathrm{d} n\left(M, z_{\text {in }}\right)}{\mathrm{d} M} b\left(M, z_{\text {in }}\right) y_{l}(M, z)\right]^{2},
\end{aligned}
$$

where $b(M, z)$ is the time-dependent linear bias factor. The matter power spectrum, $P_{\mathrm{m}}\left(k, z_{\mathrm{in}}\right)$, is related to the galaxy correlation function $P_{\mathrm{g}}\left(k, M 1, M 2, z_{\text {in }}\right)$ through the bias i.e., $P_{\mathrm{g}}\left(k, M 1, M 2, z_{\text {in }}\right)=$ $b\left(M 1, z_{\text {in }}\right) b\left(M 2, z_{\text {in }}\right) D^{2}\left(z_{\text {in }}\right) P_{\mathrm{m}}(k, z=0)$, where we adopt $b(M, z)$ given by $b(M, z)=\left(1+0.5 / \nu^{4}\right)^{0.06-0.02 n}\left(1+\left(\nu^{2}-1\right) / \delta_{\mathrm{c}}\right)(\mathrm{Jing}$ 1999). This expression for the bias factor matches accurately the results of $N$-body simulation for small haloes. This fitting formula for bias does not underestimate the clustering of small haloes with $\nu<1$, and accurately fits simulation results of CDM models and scale-free models. The difference between the fitting formula and the simulation result is generally less than $\sim 15$ per cent. In the above equation, $D(z)$ is the linear growth factor of density fluctuation, $\delta_{\mathrm{c}}=1.68$ and $\nu=\delta_{\mathrm{c}} / \sigma(M)$. In equation (6) we have utilized the Limber approximation (Limber 1954), and have set $k=l / r_{z}$, where $r_{z}$ is the comoving angular diameter distance.

\section{RESULTS AND DISCUSSIONS}

We plot the mean Compton distortion due to thermal and temperature distortion due to $\mathrm{kSZ}$ effects as a function of $z_{\text {in }}$ in Fig. 2. As can be seen from the figure, winds originating at redshifts $z \sim 6-8$ distort the CMB more than those which may have originated relatively earlier, or more recently. This can be 


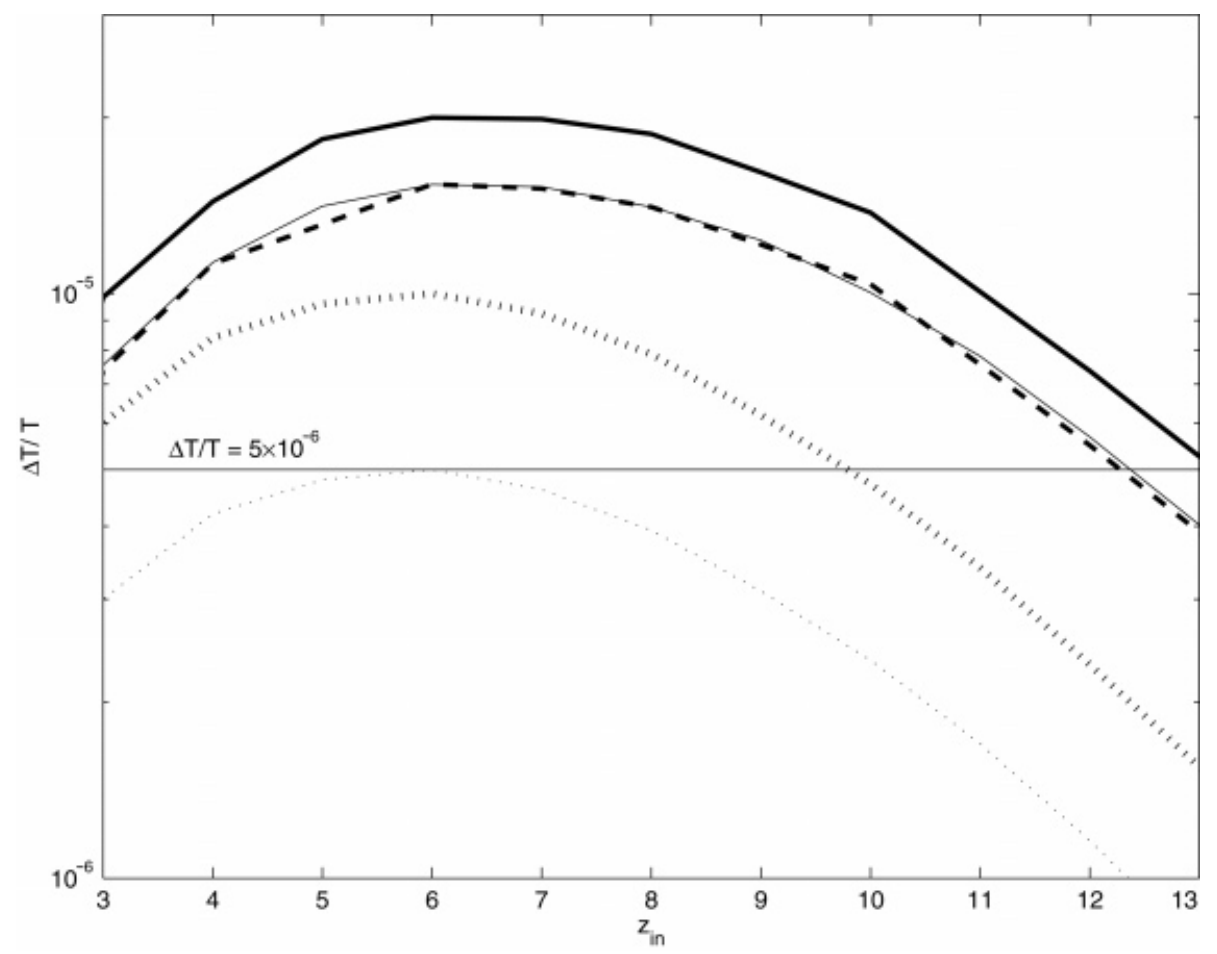

Figure 2. The temperature distortion $\Delta T / T$ (in the R-J limit) is plotted against $z_{\text {in }}$ for both $\mathrm{kSZ}$ and tSZ effects due to winds. The thick solid curve is for kSZ with $v_{r}(z=0)=400 \mathrm{~km} \mathrm{~s}^{-1}$, and the thick dashed curve is for $v_{r}(z=0)=300 \mathrm{~km} \mathrm{~s}^{-1}$. Both are with $M_{\mathrm{b}}=0.1 M$. The thin solid curve is for kSZ with $v_{r}(z=0)=400 \mathrm{~km} \mathrm{~s}^{-1}$ and $M_{\mathrm{b}}=0.05 M$. The dotted curves are for tSZ, with the solid one for $M_{\mathrm{b}}=0.1 M$ and the thin one for $M_{\mathrm{b}}=0.05 M$. A horizontal line with $\Delta T / T=5 \times 10^{-6}$ is shown for easy referencing.

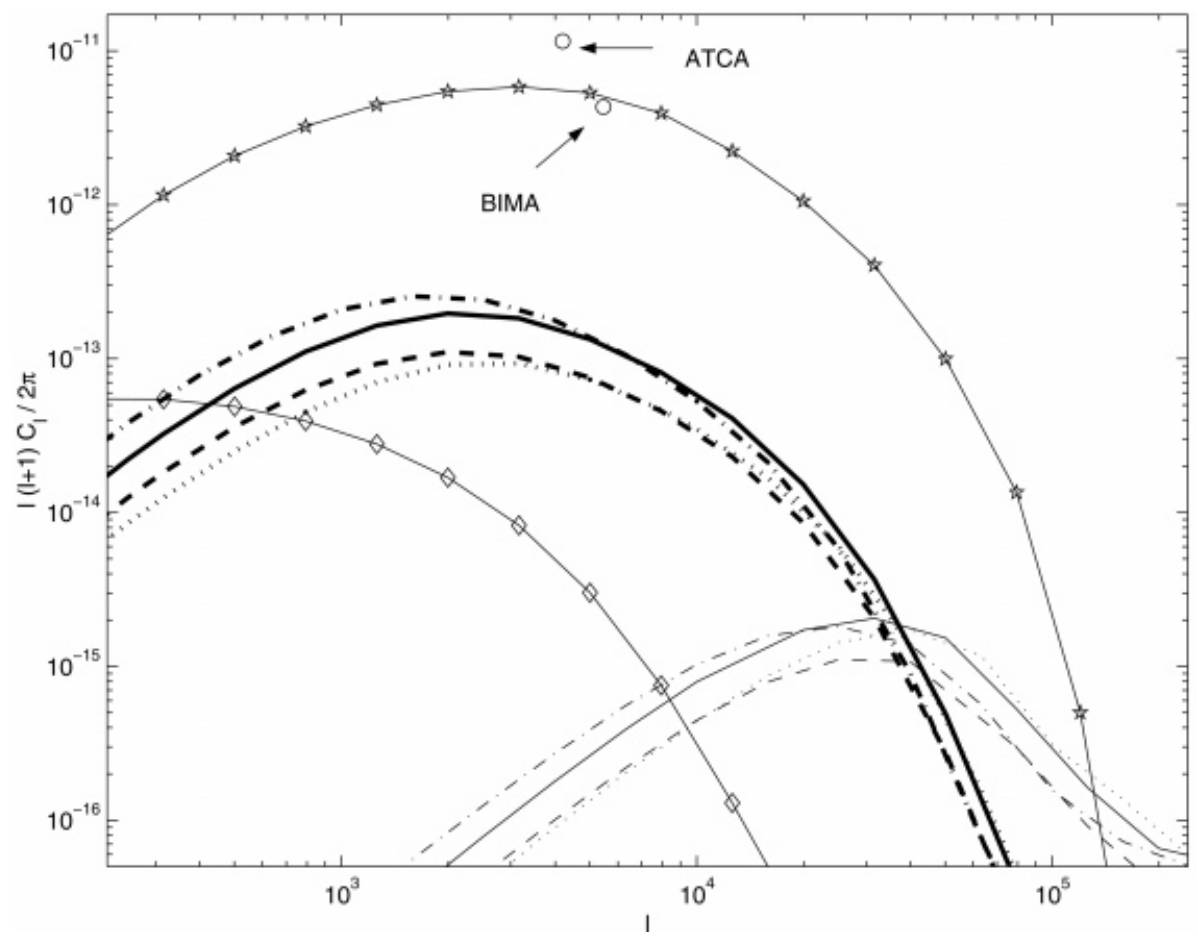

Figure 3. Angular power spectra due to the kinetic SZ effect from winds are shown for different models. The thick lines show clustering power spectra, and the thin lines show Poisson power spectra. The models shown are (1) $z_{\text {in }}=9, v_{r}(z=0)=400 \mathrm{~km} \mathrm{~s}^{-1}\left(\right.$ solid line), $(2) z_{\text {in }}=9, v_{r}(z=0)=300 \mathrm{~km} \mathrm{~s}$ (dashed line), (3) $z_{\text {in }}=7, v_{r}(z=0)=400 \mathrm{~km} \mathrm{~s}^{-1}$ (dot-dashed line), and (4) $z_{\text {in }}=11, v_{r}(z=0)=400 \mathrm{~km} \mathrm{~s}^{-1}$ (dotted line). For comparison, Poisson (solid line with stars) and clustering (solid line with squares) spectra from the thermal SZ effect from galaxy clusters are shown. We have used the same cosmological model as in Komatsu \& Kitayama (1999) for comparison (with their figs 1 and 4). The ATCA and BIMA upper limits are also marked in the figure by the two open circles. 
naively understood as follows: the distortion of the CMB due to winds is proportional to the number of galactic winds originating at $z_{\text {in }}$; the distribution of the galaxies follow from the PressSchechter formalism where the number density per comoving volume peaks at redshift $\sim 5$ for the mass range relevant here, and then falls off at higher redshifts. This combination produces the maximum distortion at an intermediate value of $z_{\text {in }}$.

We also show in Fig. 2 our results for an the case of $M_{\mathrm{b}}=$ $0.05 M$ (thin lines), which represents the case of winds with efficiencies decreased by a factor of 2 .

Next, we focus on the angular power spectrum of temperature anisotropy from galactic outflows. First, we plot in Fig. 3 the anisotropy due to the $\mathrm{kSZ}$ effect from outflows with and without clustering. We plot the power spectra for outflows for three initial redshifts, $z_{\text {in }}=7,9$ and 11 . For $z_{\text {in }}=9$, we present the results for $v_{r}(z=0)=400$ and $300 \mathrm{~km} \mathrm{~s}^{-1}$. We also plot in Fig. 3 the angular power spectra due to the tSZ effect from clusters of galaxies, with and without clustering, for comparison.

It is evident from Fig. 3 that the power spectrum due to clustering of the sources (thick lines) can be very important from CMB distortions due to winds at high redshifts. In comparison, for clusters of galaxies the Poisson power spectrum is larger than the clustering power spectrum, as shown by Komatsu \& Kitayama (1999). In that case, if flux limited clusters detected in X-ray surveys are subtracted, then the clustering power spectrum can dominate at $l<200$. In all the cases that we consider here, however, we find that the clustering power spectrum is dominant below $l \sim 3 \times 10^{4}$. This is easy to understand in the following manner. Since $C_{l}^{\text {clustering }} / C_{l}^{\text {Poisson }} \sim n(M, z) b^{2}(M, z) D^{2}(z) P_{\mathrm{m}}(k)$, with $k \sim l / r(z)$, the bias increases at high $z$ along with $r(z)$, thereby boosting up the clustering power spectrum. The evolution of bias with redshift has been studied by many authors, both theoretically (Tegmark \& Peebles 1998; Valageas, Silk \& Schaeffer 2000) and through simulations (Blanton et al. 2000; Davé et al. 2000). All of these studies show that the bias increases rapidly with increasing redshift.

The thick lines in Fig. 3 also show that the clustering spectra from winds are greater than the clustering spectrum from clusters of galaxies above $l \sim 10^{3}$ in almost all the cases we consider. The clustering spectra from winds are, however, generally smaller than the Poissonian spectrum from clusters of galaxies.

The thin lines in Fig. 3 show that the Poissonian spectra from winds (kinetic SZ) peak at $l \sim(4-6) \times 10^{4}$. Although it is swamped by the thermal SZ signature from galaxy clusters, we note that the frequency dependence of kinetic SZ effect is different from that of the thermal SZ effect. So it would be possible in principle to separate out this important signature from winds. This angular scale is an order of magnitude less than the peak for clusters of galaxies. This is beyond the ranges of observations with the upcoming PLANCK satellite, but is well within the range for $A L M A$. However, it may well be swamped by other secondary distortions of the CMB at subarcminute scales.

Recently, observational upper limits (shown in Fig. 3 by open circles) have been put on the arcminute scale distortion of the CMB temperature anisotropy by ATCA (Subrahmanyan et al. 1998) and BIMA (Holzapfel et al. 2000). In the R-J limit $\Delta T_{l}=$ $T_{\mathrm{CMB}} \sqrt{l(l+1) C_{l}}$. The angular power spectrum from any feasible model must satisfy these upper limits, in addition to the limit on the mean $y$-distortion. This has been used for the thermal SZ effect from hot gas in galaxy clusters to constrain cosmological models (Majumdar \& Subrahmanyan 2000). None of our wind models, however, violate the ATCA and BIMA limits, and hence

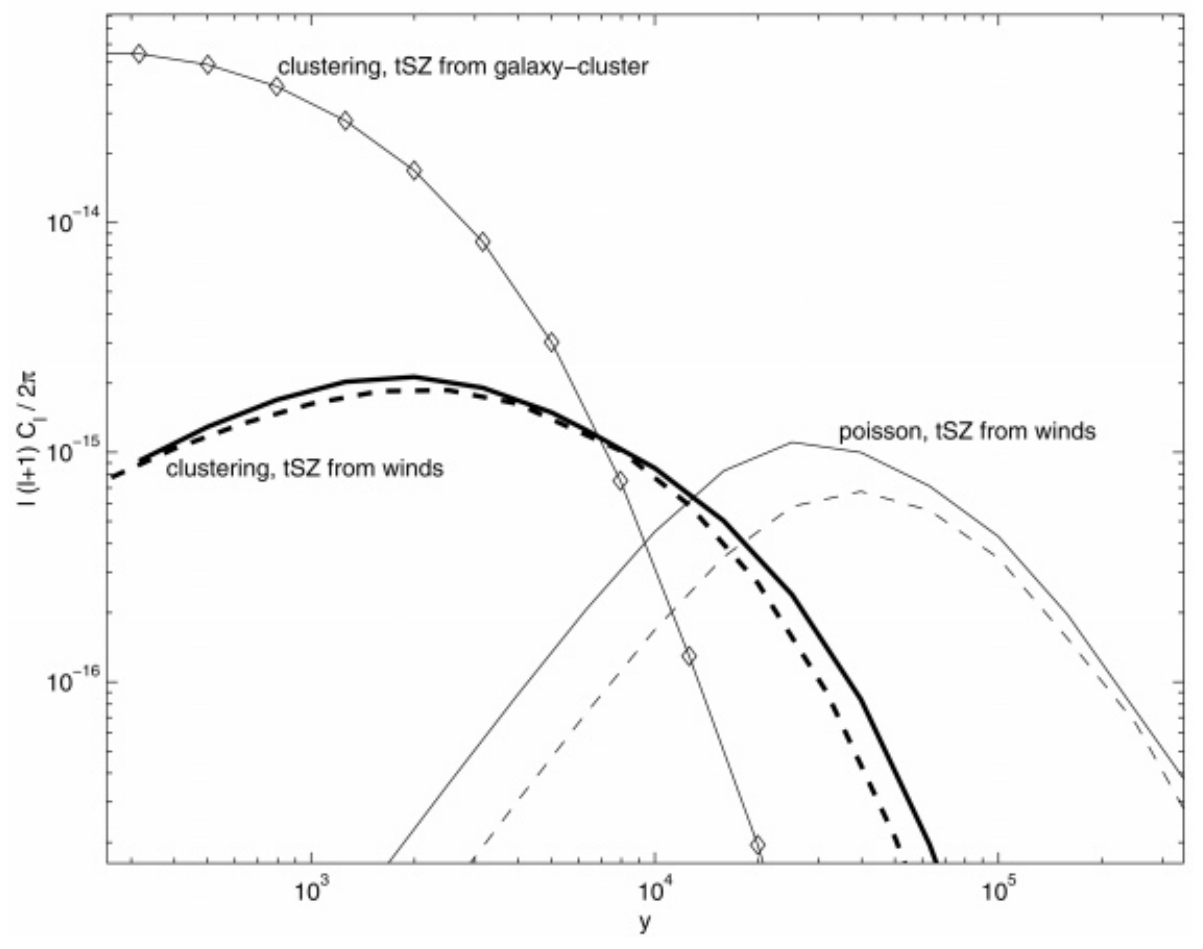

Figure 4. Angular power spectrum due to thermal SZ effect from winds are shown for different models. The thick lines show clustering power spectra, and the thin lines show Poisson power spectra. The models shown are (1) $z_{\text {in }}=9$ (solid line) and (2) $z_{\text {in }}=11$ (dashed line). For comparison, clustering (solid line with squares) spectra from the thermal SZ effect from galaxy clusters are shown. 
cannot be ruled out independent of the violation of $C O B E$ limit on $y$.

The result of the power spectrum due to clustering dominating the anisotropy brings about an interesting possibility to probe bias at high redshift. Komatsu \& Kitayama (1999) have noted that a possible detection of the clustering spectrum due to galaxy clusters would give us information on bias at high redshift. In our case, we find that the clustering spectrum due to winds (kSZ) is much larger than the clustering spectrum from galaxy clusters (tSZ), and smaller than the Poissonian spectrum from galaxy clusters (tSZ), for a wide range of $l$. If the X-ray-luminous clusters are removed, then it may be possible to detect the spectrum due to clustering of outflowing galaxies. Moreover, the different frequency dependence of $\mathrm{kSZ}$ and $\mathrm{tSZ}$ effects may allow us to separate these two effects. This may give valuable information about the evolution of bias at high redshifts, although we note that the parameters for the wind shells are not very certain, as we explain below.

We then plot the power spectra due to the tSZ effect from winds in Fig. 4. As the figure suggests, the tSZ effect is much smaller than the kSZ effect in both Poissonian and clustering cases. It is also much smaller than that from clusters of galaxies.

We compare the results with different efficiency of winds in Fig. 5, where we show the results for the cases $M_{\mathrm{b}}=0.1 M$ (thick lines) and $M_{\mathrm{b}}=0.05 M$ (thin lines). The curves show how a change of a factor of 2 in the assumption of wind efficiencies would change our results.

In Fig. 6 we have plotted the power spectrum for both tSZ and $\mathrm{kSZ}$ distortions from winds for three representative frequencies. We have chosen to show the results for 100,217 and $545 \mathrm{GHz}$. These are three of the proposed nine frequencies in which the PLANCK surveyor satellite mission would operate. The two larger frequencies would have a resolution of about 5 arcmin, and so would be able to go to $l>2000$. Note that at these $l$ values the primary anisotropy drops, and the power spectrum would be dominated by secondary anisotropies. Also, the clustering power spectra due to distortion by winds peak at these angular scales, as is evident in Fig. 3. At 100 and $545 \mathrm{GHz}$, the effect of tSZ distortion due to clusters of galaxies is greater than that from winds. However, we can take advantage of the crossover in the frequency dependence of tSZ, as given by $g(x)$, where the contribution from tSZ goes to zero. The middle panel of Fig. 6, shows the spectra at $217 \mathrm{GHz}$. At this frequency the tSZ effect from both clusters of galaxies and winds is zero, and we see that the clustering spectra due to the kSZ effect from winds exceeds the Poisson spectra from the kSZ effect from galaxy clusters. This difference is further highlighted in Fig. 7, where we plot the composite spectra (i.e., the sum of both Poisson and clustering spectra) for distortion due to galaxy clusters and winds at $217 \mathrm{GHz}$. It is clear that at this frequency, distortion due to winds peaks at $l \sim 2000-3000$ and is an order of magnitude greater than distortion due to galaxy clusters. We can thus hope to have an attempt at detecting SZ distortion due to galactic winds at high redshift with PLANCK. We also note that the anisotropies are likely to be detected and measured in the future by the proposed long-baseline interferometers such as ALMA.

The above results, however, should be viewed in the light of the uncertainties inherent in our calculation. First, the parameters of the outflows are by no means certain. The mechanical luminosity of galaxies, for example, is somewhat uncertain. The efficiency of galactic outflows, and its dependence on the mass and redshift, are also not well known. At any rate, the curves in Fig. 5 gives an idea of the magnitude of changes that might occur if some of these assumptions are changed. Lastly, our assumption for a single

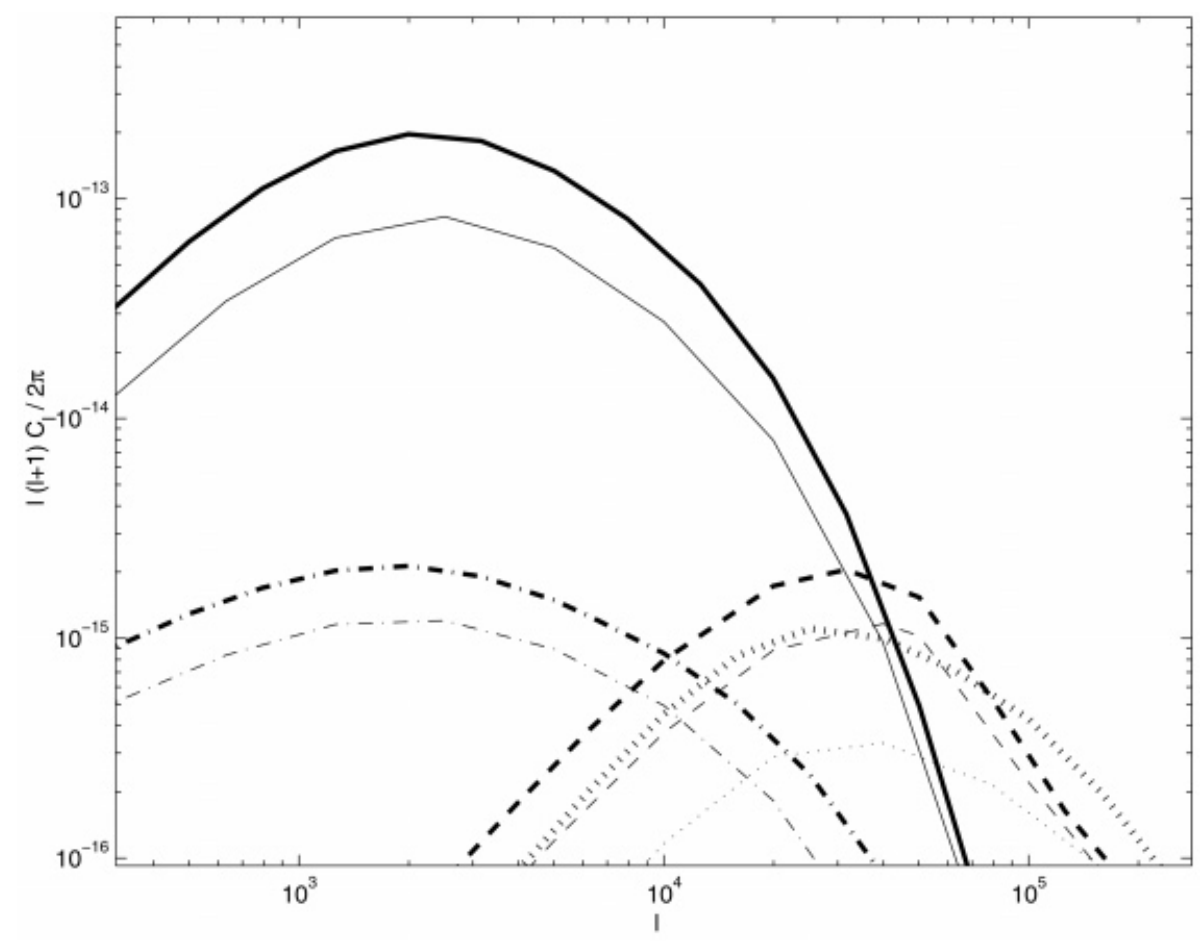

Figure 5. A comparison of the power spectrum for the two cases (i) $M_{\mathrm{b}}=0.1 M$ (thick lines) and (ii) $M_{\mathrm{b}}=0.05 M$ (thin lines) is shown, for $z_{\text {in }}=9$ and $v_{r, 0}=400 \mathrm{~km} \mathrm{~s}^{-1}$. The solid lines refer to clustering spectra due to kSZ, the dashed lines refer to the Poisson spectra for kSZ, the dot-dashed lines refer to the clustering spectra for tSZ, and the dotted lines refer to the Poisson spectra for tSZ. 

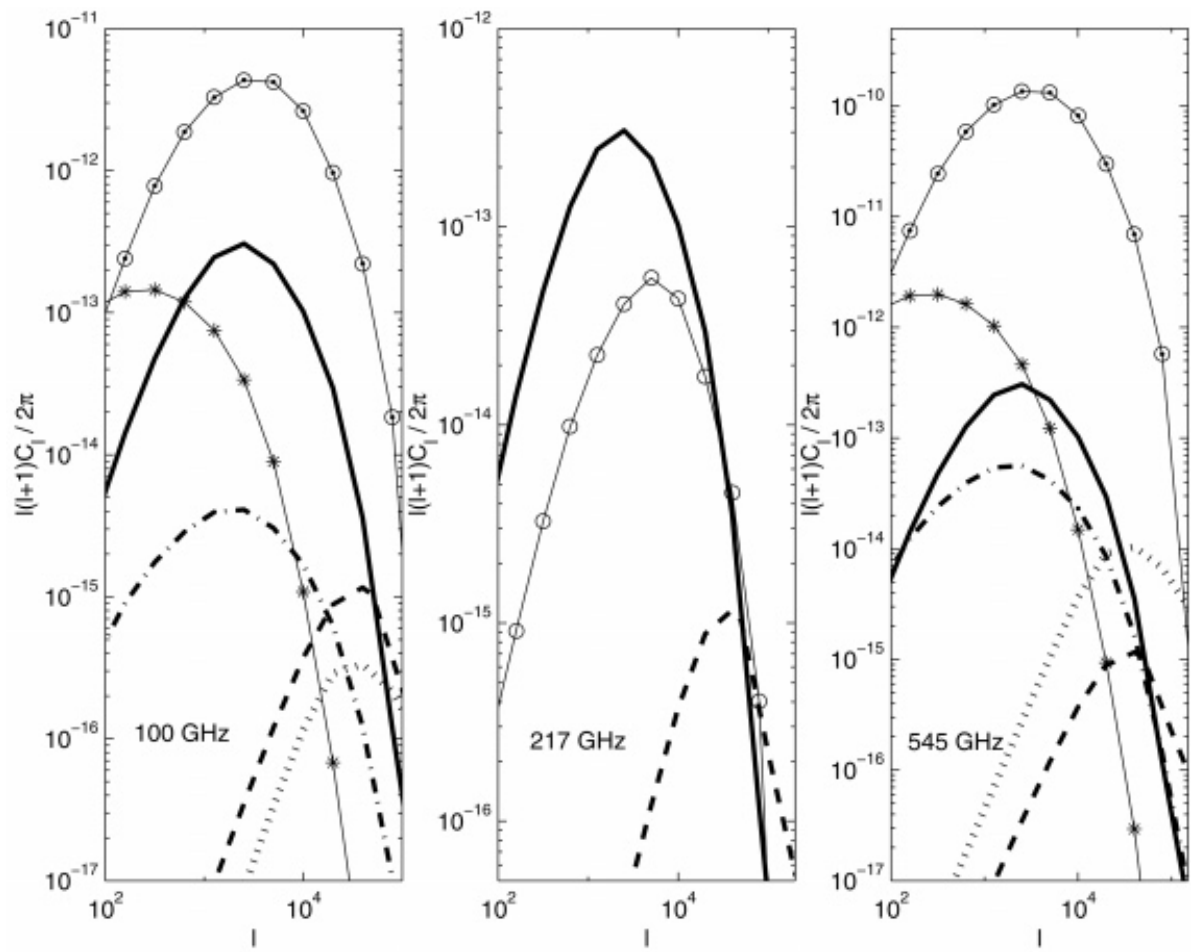

Figure 6. The figure shows the power spectrum at different frequencies. The thick lines in all the subplots are due to winds with $z_{\mathrm{in}}=9$, solid lines are for clustering spectra due $\mathrm{kSZ}$ with $v_{r, 0}=400 \mathrm{~km} \mathrm{~s}^{-1}$, and the dashed lines are the Poisson spectra for the same; the dot-dashed lines are for clustering spectra due to tSZ from winds, and the dotted lines are the Poisson spectra for the same. For comparison, in the left- and right-hand panels, Poisson spectra for tSZ from clusters (lines with filled circles) and clustering spectra for the same (lines with stars) are shown. In the middle panel, the line with open circles shows the Poisson spectra due to kSZ from galaxy clusters.

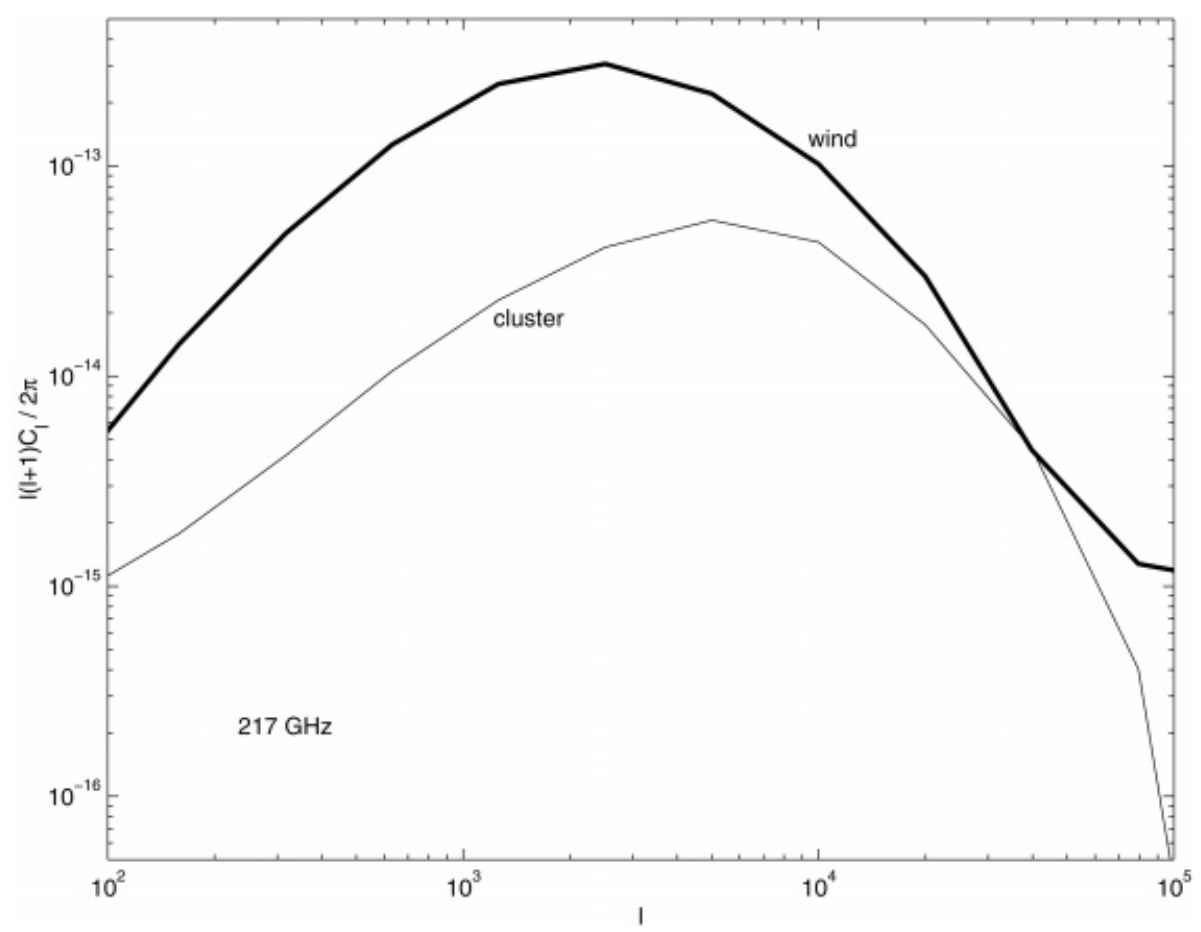

Figure 7. The composite power spectra (kSZ+tSZ, including contributions from both clustering and Poisson spectra) are shown for distortion from winds (thick line) and clusters (thin line), $z_{\text {in }}=9$ and $v_{r, 0}=400 \mathrm{~km} \mathrm{~s}^{-1}$. The spectra are calculated for the proposed PLANCK observation frequency of $217 \mathrm{GHz}$. 
epoch of galactic outflows might be naive. Also, we have assumed a mean peculiar velocity for all galaxies. Perhaps a simulation with a distribution in $z_{\text {in }}$ and $v_{r}$ would be able to address these issues in a better way.

We also note here that we have neglected the anisotropy caused by the inverse Compton cooling of the shells of outflows. Also, we have not taken into account the heating of the gas interior due to collision of the shell with the IGM. Our estimates of the anisotropy is therefore a conservative one.

Our results, however, seem to be robust with regards to the assumed upper mass limit for the outflowing galaxies, as long as the upper limit is greater than $\sim 10^{9} \mathrm{M}_{\odot}$. We note that Scannapieco \& Broadhurst (2001) have calculated the upper mass limit of outflowing galaxies in the $\Lambda \mathrm{CDM}$ universe at $z \sim 10$ to be of order $10^{9} \mathrm{M}_{\odot}$. Our results do not change significantly if we assume this upper mass cut-off instead of the one described earlier.

As far as cosmologies other than the $\Lambda \mathrm{CDM}$ are concerned, we found that the resulting anisotropy is less in the case of an SCDM ( $\Omega=1, \Lambda=0, h=0.5$ ) universe for a given $z_{\text {in }}$. This is mainly because of the fact that structures form later in an SCDM universe, although the value of $\sigma_{8}$ is larger in this case, and since the distortion in the case of wind is biased towards the high redshift. The anisotropy in the case of the OCDM universe $(\Omega=0.35$, $\Lambda=0, h=0.65$ ) is slightly less than in the $\Lambda \mathrm{CDM}$ universe. There are two reasons for this: in the OCDM universe, first, the $C O B E$-normalized $\sigma_{8}$ for OCDM is much less than in $\Lambda \mathrm{CDM}$ case and, secondly, peculiar velocities are also smaller at high redshift, for a given normalization at the present epoch.

\section{CONCLUSIONS}

We have calculated the SZ distortion of the CMB due to galactic winds at high redshift, originating at a single epoch $z_{\text {in }}$, from galaxies of masses between $5 \times 10^{7}$ and $10^{11} \mathrm{M}_{\odot}$, in a $\Lambda \mathrm{CDM}$ universe. We summarize our findings below.

(1) We confirm the previous estimates of the mean $y$-distortion due to the thermal SZ effect. We, however, found the kinetic SZ effect to be more important than the thermal SZ effect in terms of the effect on the angular power spectrum of distortions.

(2) We obtained the angular power spectrum of distortions with and without clustering of parent galaxies. The power spectrum due to the kinetic SZ effect (Poisson) is found to be comparable or larger than the thermal SZ effect from clusters of galaxies for $l \gtrsim 10^{5}$.

(3) We found that clustering of low-mass galaxies at high redshift could increase the angular power spectum of distortions. The power spectrum due to clustering of parent galaxies of outflows (kinetic SZ) was found to be also larger than the clustering power spectrum from galaxy clusters (thermal SZ) and somewhat smaller than the Poisson power spectrum from galaxy clusters (thermal SZ). We have explicitly shown the frequency dependence of the various power spectra. It is possible that the clustering power spectrum from winds can be estimated after subtracting X-ray-luminous clusters dominating the Poisson power spectrum from clusters, or by using the different frequency dependence for thermal and kinetic SZ effects, yielding information on bias of low-mass galaxies at high redshifts.

(4) We have shown how the total power spectra (kinetic and thermal SZ, including both Poisson and clustering effects) for winds will dominate over the corresponding spectra for clusters at $217 \mathrm{GHz}$, a proposed frequency for the PLANCK satellite mission.
We conclude that the SZ distortion from galactic winds at high redshift, if present, could constitute an important source of secondary CMB anisotropy on arcminute and subarcminute scales.

\section{ACKNOWLEDGMENTS}

We thank the referee, Priyamvada Natarajan, for her detail comments which have improved the paper considerably. SM thanks staff at the Raman Research Institute, Bangalore, for hospitality and for providing computational facilities. BBN and MC acknowledge a grant from the India-Japan Cooperative Research Programme for partial support of this work, and BBN acknowledges the hospitality of the National Astronomical Observatory, Tokyo. SM also thanks Pijush Bhattacharjee for much encouragement.

\section{REFERENCES}

Aghanim N., Desert F. X., Puget J. L., Gispert R., 1996, A\&A, 311, 1

Aghanim N., Balland C., Silk J., 2000, A\&A, 357, 1

Bahcall N. A., Oh S. P., 1996, ApJ, 462, L49

Bahcall N. A., Cen R., Gramann M., 1994, ApJ, 430, L13

Bardeen J. M., Bond J. R., Kaiser N., Szalay A. S., 1986, ApJ, 304, 15

Blanton M., Cen R., Ostriker J. P., Strauss M. A., Tegmark M., 2000, ApJ, 531,1

Bunn E., White M., 1997, ApJ, 480, 6

Caroll S. M., Press W. H., Turner E. L., 1992, ARA\&A, 30, 499

Ciardi B., Ferrara A., Governato F., Jenkins A., 2000, MNRAS, 314, 611

Cole S., Kaiser N., 1988, MNRAS, 233, 637

Davé R., Hernquist L., Katz N., Weinberg D. H., 1999, in Mazure A., Le Fèvre O., Le Brun V., eds, ASP Conf. Ser. Vol. 200, Clustering at High Redshift. Astron. Soc. Pac., San Francisco

Dekel A., Silk J., 1986, ApJ, 303, 39

Ferrara A., Pettini M., Shchekinov Y., 2000, MNRAS, 319, 539

Franx M., Illingworth G., Kelson D., van Dokkum P., Tran K.-V., 1997, ApJ, 486, L75

Gnedin N. Y., Ostriker J. P., 1997, ApJ, 486, 581

Gramann M., 1998, ApJ, 493, 28

Haiman Z., Rees M., Loeb A., 1997, ApJ, 476, 458 [erratum 484, 985 (1997)]

Heckman T., 1997, Rev. Mex. Astron. Astrofis., 6, 156

Heckman T. M., Armus L., Miley G. K., 1990, ApJS, 74, 833

Holzapfel W. L., Carlstrom J. E., Grego L., Holder G., Joy M., Reese E. D., 2000, ApJ, 539, 57

Jing Y. P., 1999, ApJ, 515, L45

Komatsu E., Kitayama T., 1999, ApJ, 526, L1

Lahav O., Lilje P. B., Primack J. R., Rees M., 1991, MNRAS, 251, 136

Larson R. B., 1974, MNRAS, 166, 585

Limber D., 1954, ApJ, 119, 655

Mac Low M. M., Ferrara A., 1999, ApJ, 513, 142

Majumdar S., Subrahmanyan R., 2000, MNRAS, 312, 724

Matteucci F., Tornambé A., 1987, A\&A, 185, 51

Miralda-Escudé J., Rees M. J., 1997, ApJ, 478, L57

Molnar S. M., Birkinshaw M., 2000, ApJ, 537, 542

Moscardini L., Branchini E., Brunozzi P. T., Borgani S., Plionis M., Coles P., 1996, MNRAS, 282, 384

Natarajan P., Sigurdsson S., 1999, MNRAS, 302, 288

Natarajan P., Sigurdsson S., Silk J., 1998, MNRAS, 298, 577

Nath B., Trentham N., 1997, MNRAS, 291, 505

Peacock J. S., Heavens A. F., 1990, MNRAS, 243, 133

Peebles P. J. E., 1980, Large Scale Structure of the Universe. Princeton Univ. Press, Princeton

Pettini M., Kellogg M., Steidel C., Dickinson K. E., Adelberger K. L., Giavalisco M., 1998, ApJ, 508, 539

Press W. H., Schechter P., 1974, ApJ, 187, 425 
Rauch M. et al., 1998, ApJ, 489, 1

Rephaeli Y., 1995, ARA\&A, 33, 541

Saito M., 1979, PASJ, 31, 181

Scannapieco E., Broadhurst T., 2001, 549, 28

Sheth R. K., Tormen G., 1999, MNRAS, 308, 119

Songaila A., 1997, ApJ, 490, L1

Songaila A., Cowie L. L., 1996, AJ, 112, 335

Subrahmanyan R., Kesteven M. J., Ekers R. D., Sinclair M., Silk J., 1998, MNRAS, 298, 1189

Sugiyama N., 1995, ApJS, 100, 281

Sunyaev R. A., Zel'dovich Ya.B., 1972, Comments Astrophys. Space Phys., 4, 173
Tegmark M., Peebles P. J. E., 1998, ApJ, 500, L79

Tegmark M., Silk J., Evrard A., 1993, ApJ, 417, 54 (TSE)

Thoul A., Weinberg D., 1996, ApJ, 465, 608

Valageas P., Silk J., Schaeffer R., 2001, A\&A, 366, 363

Weinberg D. H., Miralda-Escudé J., Hernquist L., Katz N., 1997, ApJ, 490, 564

White D. M., Navarro J. F., Evrard A. E., Frenk C. S., 1993, Nat, 366, 429

This paper has been typeset from a TEX/LTEX file prepared by the author. 